\title{
LncRNA-HEIH is a Novel Diagnostic and Predictive Biomarker in Gastric Cancer
}

\author{
Xin Chen, ${ }^{1, *}$ Xue Sun, ${ }^{2, *} \mathrm{Xi} \mathrm{Li}^{3}, \mathrm{Lu} \mathrm{Xu},{ }^{1}$ and Wenyan $\mathrm{Yu}^{4}$
}

Background: Gastric cancer (GC) is associated with a high mortality rate. Long noncoding RNA (lncRNA)high expression in hepatocellular carcinoma $(\mathrm{HEIH})$ has recently gained interest as a marker for the detection of several cancer types. This study was designed to uncover the function of lncRNA-HEIH in GC.

Materials and Methods: Oncomine was used to analyze HEIH expression in cancerous and paired noncancerous tissues of GC patients. Subsequently, the expression levels of HEIH in GC cells was determined by reverse transcription-quantitative polymerase chain reaction (RT-qPCR). In addition, the effects of HEIH expression level on clinicopathological parameters and prognosis were further studied by statistical analysis and Kaplan-Meier survival curves. GC cell proliferation and the influence of HEIH on the sensitivity of cells to oxaliplatin following HEIH knockdown were assessed using sulforhodamine blue (SRB) assays in the MKN45 and AGS cell lines. In addition, the expression levels of p53 were detected by RT-qPCR following knockdown of HEIH.

Results: The lncRNA-HEIH was highly expressed in both GC tissues and GC cell lines. Patients with high HEIH expression were associated with medium-high differentiation $(p=0.0058)$, distant metastasis $(\mathrm{M}$, $p=0.0378)$, lymph node metastasis $(\mathrm{N}, p=0.0083)$, and a deeper tumor invasion $(\mathrm{T}, p=0.0204)$. Elevated expression levels of HEIH in GC patients were associated with a worse prognosis compared to GC patients with low HEIH expression. This finding was supported by the parameters of overall survival $(p=3.3 \mathrm{e}-06)$, first progression $(p=0.00028)$, and postprogression $(p=1.5 \mathrm{e}-08)$. Downregulation of HEIH expression inhibited cell proliferation, enhanced oxaliplatin sensitivity, and induced the expression of p53 in MKN45 and AGC cells.

Conclusion: These findings provide evidence that HEIH may be useful as a prognostic biomarker in GC. This lncRNA may also serve as a potential therapeutic target in GC patients.

Keywords: lncRNA-HEIH, gastric cancer, oncomine, proliferation, p53

\section{Introduction}

G ASTRIC CANCER (GC) is the fifth most frequently diagnosed malignant tumor and exhibits the third highest cancer-related mortality rate worldwide (Smyth et al., 2020). Approximately $80 \%$ of patients with GC in China are diagnosed at an advanced stage (Zong et al., 2016). The disease is accompanied by regional lymph node invasion and distant metastasis (Rawla and Barsouk, 2019). Emerging evidence indicates that the 5-year overall survival (OS) rate for patients with metastatic cancer following diagnosis is only 5\% (Thrift and El-Serag, 2020). Chemotherapy is recognized as the standard first-line treatment for patients with advanced GC, including fluoropyrimidine and oxaliplatin (Wang et al., 2019). Despite improvements in chemotherapeutic options, patients with GC often exhibit poor prognosis due to drug resistance and postoperative recurrence (Zhang et al., 2016). Consequently, the exploration of novel biomarkers that can be used to assess the prognosis of GC is particularly significant for effective clinical decision making.

Long noncoding RNAs (lncRNAs) comprise a class of noncoding RNAs with $\sim 200$ nucleotides in length (Iyer et al., 2015). lncRNAs are involved in cellular development and differentiation (Spizzo et al., 2012; Fatica and Bozzoni, 2014). IncRNA high expressed in hepatocellular carcinoma (HEIH) is a member of the lncRNA family, which was initially reported and investigated in hepatocellular carcinoma (Yang et al., 2011). Moreover, the tumor-promoting role of HEIH in multiple carcinoma types has been reported, including non-small cell lung cancer (Jia et al., 2019), melanoma

Departments of ${ }^{1}$ General Surgery and ${ }^{2}$ Emergency Intensive Care Unit, The First Affiliated Hospital of Soochow University, Suzhou, China.

${ }^{3}$ Department of Technologies, Burning Rock Biotech, Guangzhou, China.

${ }^{4}$ Department of Oncology, The First Affiliated Hospital of Soochow University, Suzhou, China.

*These authors contributed equally to this work. 
(Zhao et al., 2017), and breast cancer (Li et al., 2019). However, the association between lncRNA-HEIH and GC has not been previously studied.

Based on the results of previous studies, the expression levels of HEIH were investigated in GC tissues and cell lines and the clinical parameters and prognostic value of $\mathrm{HEIH}$ were analyzed in patients with GC. Cell viability and the function of HEIH in mediating oxaliplatin sensitivity in GC were explored by cell proliferation assays. The effect of HEIH on the expression of p53 was also investigated in GC samples. This study aimed to provide novel information on the vital role of HEIH in GC.

\section{Materials and Methods}

\section{Clinical samples}

A total of $215 \mathrm{GC}$ patient tissue specimens, including 125 samples from male subjects and 90 from female subjects with an age range from 37 to 86 years, were obtained from the Department of General Surgery, The First Affiliated Hospital of Soochow University (Suzhou, China). The clinical parameters were collected, including gender (male/female), age $(<60 / \geq 60$ years old), differentiation (medium-high/low), pathological depth of invasion stage $(\mathrm{T} 1+2 / \mathrm{T} 3+4)$, pathological lymph node metastasis stage (-/+) and distant metastasis stage $\left(\mathrm{M}_{0} / \mathrm{M}_{1}\right)$. The inclusion criteria were as follows: (1) histopathological diagnosis of adenocarcinoma; (2) patients who had not received antitumor treatment before the study; and (3) computed tomography of the chest, abdomen, and pelvis did not show evidence of distant metastasis. The exclusion criteria were as follows: (1) patients who cannot tolerate surgical treatment and (2) patients who did not allow specimen provision. All the patients involved in this study signed informed consent agreements. The protocol of this study was approved by the Ethics Committee of the First Affiliated Hospital of Soochow University (Suzhou, China; approval no. 2021015). None of the patients had received chemotherapy or radiotherapy before surgery.

\section{Oncomine database analysis}

The data were derived from the Oncomine database, an online cancer microarray/gene database for RNA and DNA sequences, and were used to analyze HEIH expression in GC and normal tissues by the $t$-test. The cut-off $p$-value and fold change were as follows: $p$-value $\leq 0.0001$ and fold change: 2 .

\section{Cell culture}

The normal gastric epithelial cell line GES1 and the human GC cell lines (MKN45, NCl-N87, AGS, and HGC-27) were provided by the Cell Bank of the Chinese Academy of Science (Shanghai, China). The cells were cultured in RPMI1640 medium (Gibco, Carlsbad, CA) supplemented with $10 \%$ fetal bovine serum (FBS; Invitrogen, Carlsbad, CA) at $37^{\circ} \mathrm{C}$ in a humidified atmosphere containing $5 \% \mathrm{CO}_{2}$.

\section{Reverse transcription-quantitative polymerase chain reaction}

Total RNA was extracted from GC cells using the TRIzol reagent (Invitrogen) according to the manufacturer's in- structions. Subsequently, cDNA was amplified by reverse transcription-quantitative polymerase chain reaction (RTqPCR) using SYBR Premix Ex Taq kit (TaKaRa, Tokyo, Japan). The following thermal cycling conditions were used for the PCR: $95^{\circ} \mathrm{C}$ for $30 \mathrm{~s} ; 45$ cycles of $95^{\circ} \mathrm{C}$ for $5 \mathrm{~s}$; and $60^{\circ} \mathrm{C}$ for $30 \mathrm{~s}$. The relative mRNA expression of the target genes was calculated by the $2^{-\Delta \Delta \mathrm{Cq}}$ method. GAPDH was used as an internal control and all samples were run in triplicate. The primer sequences used were as follows: IncRNAHEIH: forward CCTCTTGTGCCCCTTTCTT and reverse ATGGCTTCTCGCATCCTAT; p53: forward TAACAGTT CCTGCATGGGCGGC and reverse AGGACAGGCACAA ACACGCACC; and GAPDH: forward TTCCTGGGCATG GAGTCCT and reverse TGATCTTCATTGTGCTGGGTG.

\section{Kaplan-Meier plotter survival analysis}

The prognostic value of HEIH mRNA expression in GC was evaluated by an online database, the Kaplan-Meier (KM) plotter. The parameters OS, first progression (FP), and postprogression survival (PPS) were assessed. The patient samples were divided into two groups (high vs. low expression) based on the median values of HEIH mRNA expression. The corresponding survival curve, hazard ratios (HR), 95\% confidence intervals, and $p$-values were calculated and plotted in $\mathrm{R}$. The log-rank test was also performed and a $p<0.05$ was considered for significant differences.

\section{Small interfering RNA transfection}

MKN45 and AGS cells were seeded into six-well plates (Corning, Acton, MA) and left to grow to $\sim 80 \%$ confluence. siHEIH-1 (siRNA-1, 5'-TGC GCC TTC CCT CTA ACC TTA ATT CAA GAG ATT AAG-3'), siHEIH-2 (siRNA-2, 5'-TGG CAA GAT GAA CGT CTG AAA TTT CAA GAG AAT TTC- $3^{\prime}$ ), and negative control (NC, all Genepharma, Shanghai, China) sequences were transfected into the cells. The small interfering RNA (siRNA) was transfected into MKN45 and AGS cells using Lipofectamine2000 (Invitrogen) according to the manufacturer's instructions.

\section{Oxaliplatin treatment}

Oxaliplatin (Selleck, Shanghai, China) was added at the indicated concentrations $(12.5,25,50,100$, and $200 \mu \mathrm{M})$ into the culture medium of (siHEIH-) MKN45 and (siHEIH-) AGS cells. The cells were incubated with oxaliplatin for $48 \mathrm{~h}$ at $37^{\circ} \mathrm{C}$ in the presence of $5 \% \mathrm{CO}_{2}$.

\section{Cell proliferation assay}

Cell proliferation was determined by the sulforhodamine blue (SRB) assay (Orellana and Kasinski, 2016). The cells were seeded into a 96-well plate (Corning) for the indicated time points $\left(1,2\right.$, and 3 days) with $1 \times 10^{4}$ cells $(100 \mu \mathrm{L}$ per well). The cells were fixed with $50 \mu \mathrm{L} \mathrm{30 \%} \mathrm{(v/v)} \mathrm{trichloro-}$ acetic acid (TCA; Sinopharm Reagent, Shanghai, China) for $60 \mathrm{~min}$ at $4^{\circ} \mathrm{C}$ and washed five times using distilled water. Following washing and fixation, the cells were stained with $100 \mu \mathrm{L} 0.4 \%(\mathrm{w} / \mathrm{v})$ sulforhodamine B (SRB, prepared in $1 \%$ acetic acid) (Sigma-Aldrich, St. Louis, MO) for $60 \mathrm{~min}$ at room temperature. The wells were rinsed with $1 \%$ acetate acid solution and air dried. The stain was solubilized 

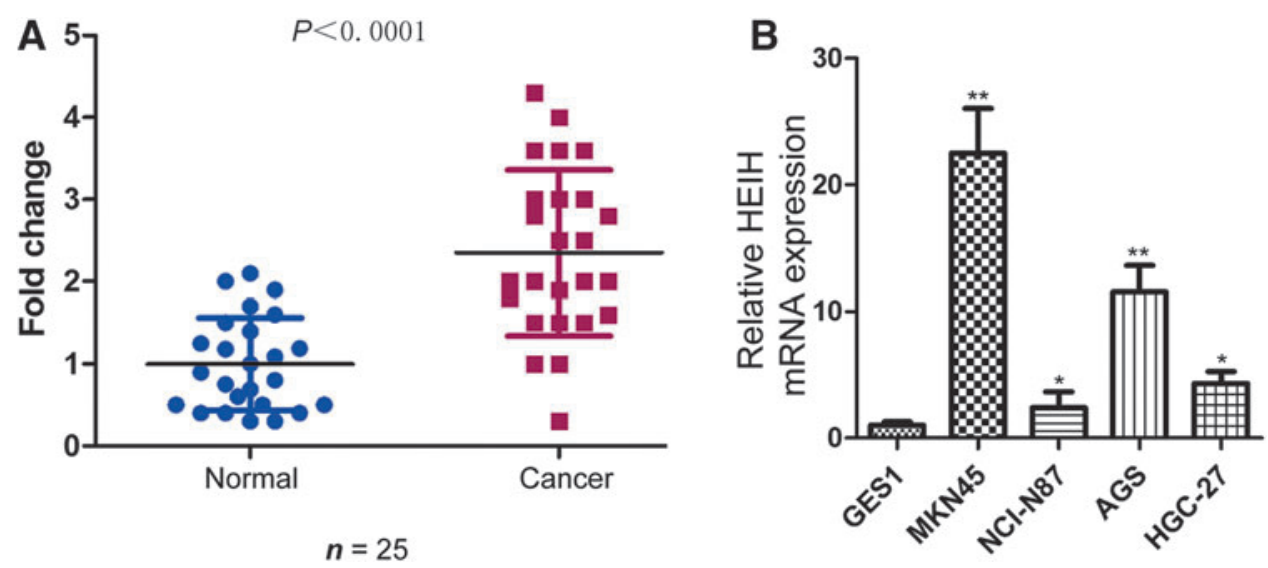

FIG. 1. (A) The lncRNA-HEIH expression levels were assessed in 25 tumor and adjacent nontumor tissues from Oncomine $(p<0.0001)$. (B) The lncRNA-HEIH mRNA expression levels were evaluated in GC and normal gastric epithelial cell lines by RTqPCR. $* * p<0.01$ and $* p<0.05$ versus GES1. GC, gastric cancer; lncRNA-HEIH, long noncoding RNA high expressed in hepatocellular carcinoma; RT-qPCR, reverse transcription-quantitative polymerase chain reaction. Color images are available online.

in $100 \mu \mathrm{L} 10 \mathrm{mM}$ Tris $(\mathrm{pH} 10.5)$. The absorbance was recorded with a spectrometer at $490 \mathrm{~nm}$ (Infnite M200, Tecan, Austria).

\section{Statistical analysis}

The GraphPad Prism 7.0 software was used for statistical analysis. The data were processed by $t$-test and presented as mean \pm standard deviation. The association between HEIH expression and clinicopathological parameters was measured by the $\chi^{2}$ test. The differences were considered significant at $p<0.05$.

\section{Results}

\section{High expression of HEIH in GC tissues and cell lines}

The mRNA expression levels of HEIH in GC and normal GC tissues were determined in a cohort of 25 patients from the Oncomine database. The results revealed that HEIH mRNA levels were significantly higher in $\mathrm{GC}$ tissues than those in normal tissues $(p<0.0001$; Fig. 1A).

In addition, higher expression levels of HEIH were observed in the human GC cell lines (MKN45, NCl-N87, AGS, and HGC-27) compared with those noted in the normal gastric epithelial cell line GES1, as determined by RT-qPCR $(p<0.05$, Fig. 1B). Finally, the MKN45 and AGS cell lines were selected for subsequent studies.

\section{High HEIH expression levels are associated with more aggressive clinicopathological characteristics in GC patients}

A total of 215 GC patients were selected, which included 125 male patients and 90 female patients. The association between HEIH expression levels and GC patient clinicopathological parameters is shown in Table 1. A significant difference was noted between the expression levels of $\mathrm{HEIH}$ and the $\mathrm{T}, \mathrm{N}$, and $\mathrm{M}$ stage. High expression levels of HEIH were significantly associated with medium-high differentiation $(p=0.0058)$,
$\mathrm{M}$ stage $(p=0.0378), \mathrm{N}$ stage $(p=0.0083)$, and $\mathrm{T}$ stage $(p=0.0204)$. The parameters gender and age were not associated with high HEIH expression levels $(p<0.05)$.

High HEIH expression predicts poor survival in GC patients

Patients with GC and elevated HEIH mRNA expression levels exhibited decreased OS $(\mathrm{HR}=1.86 ; p=3.3 \mathrm{e}-06$;

Table 1. The Association Between Long NONCODING RNA HigH EXPRESSED

in Hepatocellular Carcinoma Expression and Clinicopathological Characteristics of Patients With Gastric Cancer $(n=215)$

\begin{tabular}{lcccc}
\hline & & \multicolumn{2}{c}{ HEIH levels } & \\
\cline { 3 - 4 } Variable & $\begin{array}{c}\text { Patients } \\
(\mathrm{n})\end{array}$ & $\begin{array}{c}\text { Low } \\
(\mathrm{n}=64)\end{array}$ & $\begin{array}{c}\text { High } \\
(\mathrm{n}=151)\end{array}$ & $\mathrm{p}$ \\
\hline Gender & & & & \\
$\quad$ Male & 125 & 33 & 92 & 0.2031 \\
$\quad$ Female & 90 & 31 & 59 & \\
Age & & & & \\
$\quad<60$ & 121 & 36 & 85 & 0.9955 \\
$\quad \geq 60$ & 94 & 28 & 66 & \\
Differentiation & & & & \\
$\quad$ Medium-high & 100 & 39 & 61 & 0.0058 \\
$\quad$ Low & 115 & 25 & 90 & \\
M & & & & \\
$\quad$ M0 & 165 & 55 & 110 & 0.0378 \\
$\quad$ M1 & 50 & 9 & 41 & \\
$\mathrm{~N}$ & & & & \\
$\quad-$ & 82 & 33 & 49 & 0.0083 \\
$\quad+$ & 133 & 31 & 102 & \\
T & & & & \\
T1+2 & 95 & 36 & 59 & 0.0204 \\
T3+4 & 120 & 28 & 92 & \\
\hline
\end{tabular}

$\mathbf{M}_{0}$, no distant metastasis; $\mathbf{M}_{1}$, distant metastasis; $\mathrm{N}(-)$, no lymph node metastasis; $\mathrm{N}(+)$, lymph node metastasis; T, depth of invasion.

$\mathrm{HEIH}$, high expressed in hepatocellular carcinoma. 
Overall survival

A

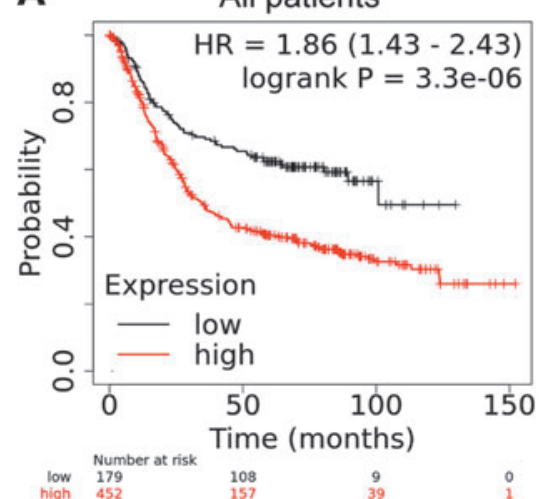

Female

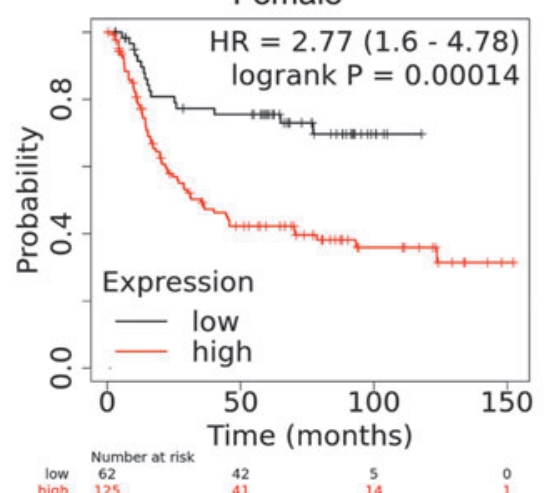

Stage III
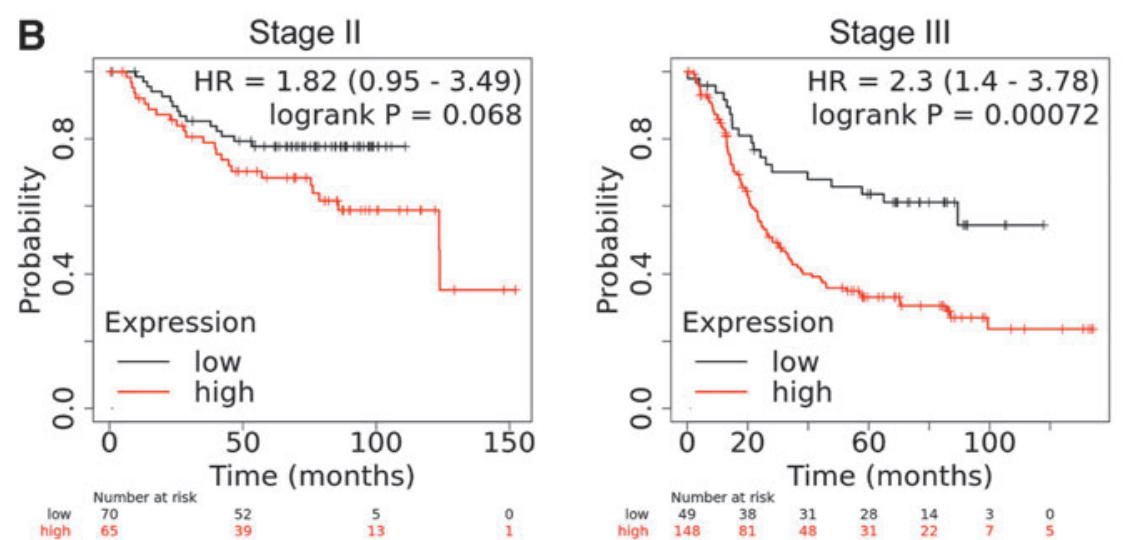

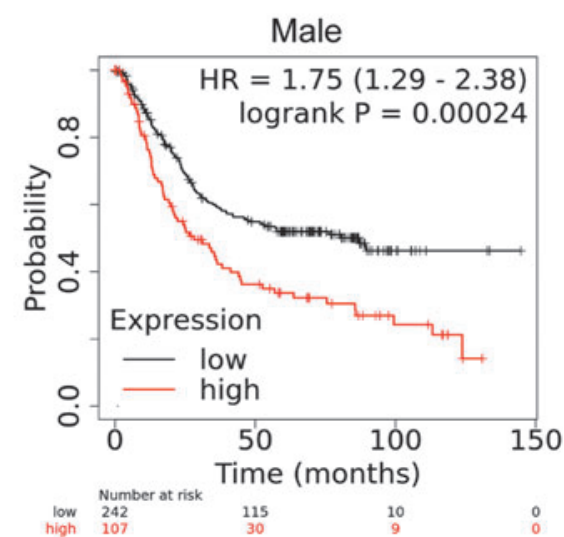

Stage IV

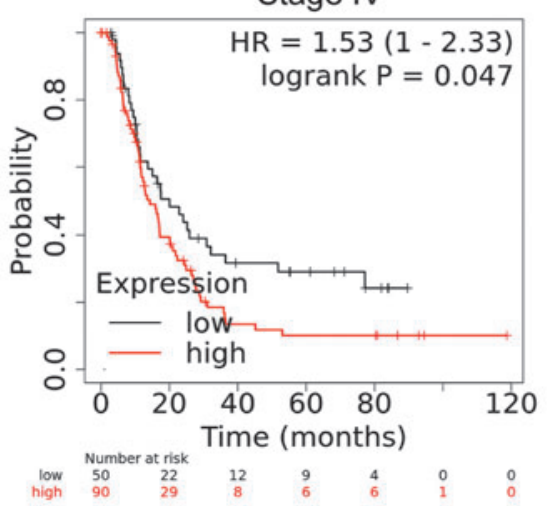

C Surgery alone
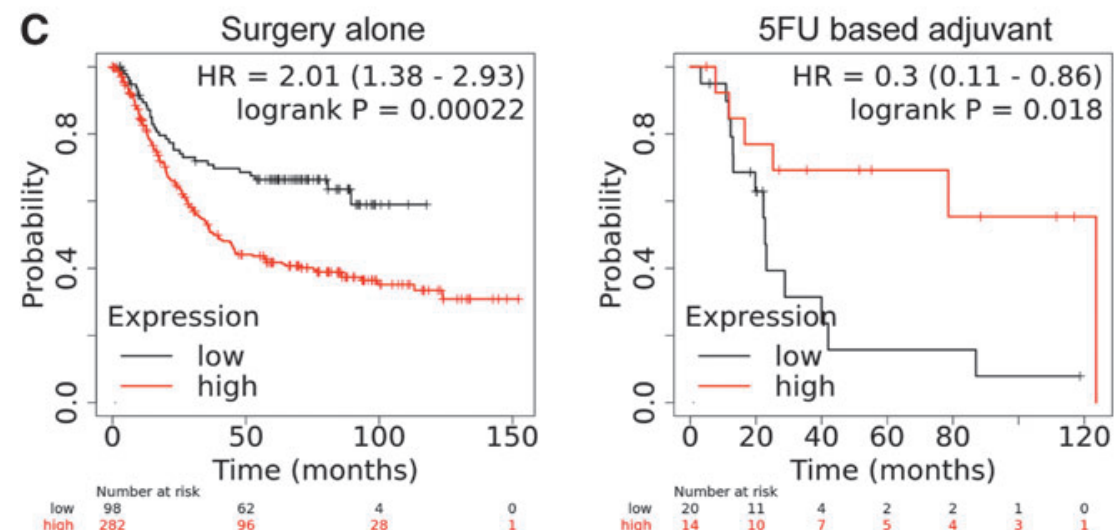

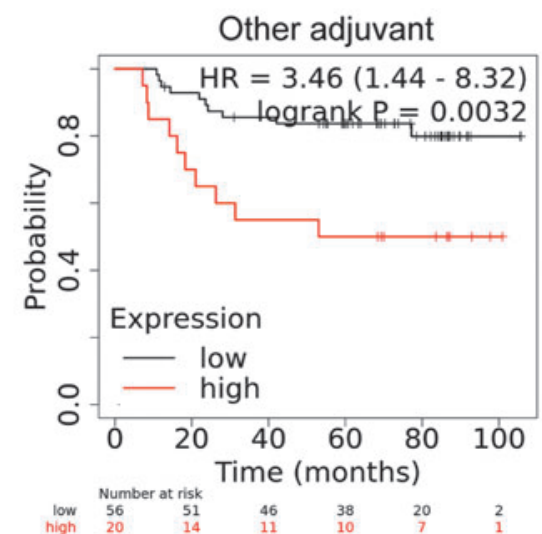

FIG. 2. The prognostic values of IncRNA-HEIH in GC. (A) High mRNA levels of HEIH were associated with lower OS in all GC patients (female or male). (B) High mRNA levels of HEIH were associated with lower OS in stage III and IV, but not in stage II GC patients. (C) High mRNA levels of HEIH were associated with OS in GC patients who received single treatment with surgery, 5FU-based adjuvant therapy and other adjuvant therapy. OS, overall survival. Color images are available online.

Fig. 2A), FP (HR=1.71, $p=0.00028$; Fig. 3A), and PPS $(\mathrm{HR}=2.17, p=1.5 \mathrm{e}-08$; Fig. $4 \mathrm{~A})$. In addition, the prognosis of patients with high and low HEIH expression levels and different gender and stage (II, III, IV) was assessed according to the treatment selection, which included surgical treatment, 5FU-based adjuvant, and other adjuvant therapies. The statistical analysis of the variables demonstrated the following results: female subjects (OS, $\mathrm{HR}=2.77, p=0.00014$; FP, $\mathrm{HR}=2.57, p=3 \mathrm{e}-06$; and PPS, $\mathrm{HR}=3.1, p=5.7 \mathrm{e}-05$ ), male subjects $\quad(\mathrm{OS}, \mathrm{HR}=1.75, p=0.00024 ; \mathrm{FP}, \mathrm{HR}=1.53$, $p=0.00052 ;$ and PPS, HR $=2.12, p=6 \mathrm{e}-06)$, stage II (PPS, $\mathrm{HR}=2.9, p=0.00098)$, stage III (OS, HR $=2.3, p=0.00072$; $\mathrm{FP}, \mathrm{HR}=2.14, p=0.0012$; and $\mathrm{PPS}, \mathrm{HR}=2.36, p=8.1 \mathrm{e}-05$ ), stage IV (OS, HR $=1.53, p=0.047$, and PPS, HR $=1.89$, $p=0.0074)$, patients receiving surgical treatment alone (OS, HR=2.01, $p=0.00022$; FP, $\mathrm{HR}=1.79, p=0.0016$; and PPS, HR=2.09, $p=2.3 \mathrm{e}-06$ ), 5FU-based adjuvant (OS, $\mathrm{HR}=0.3, p=0.018$ ), and other adjuvant (OS, $\mathrm{HR}=3.46$, $p=0.00032$; FP, $\mathrm{HR}=3.52, p=0.00081 ;$ and PPS, $\mathrm{HR}=3.02$, $p=0.013)$. Moreover, the data indicated that high HEIH 
First progression
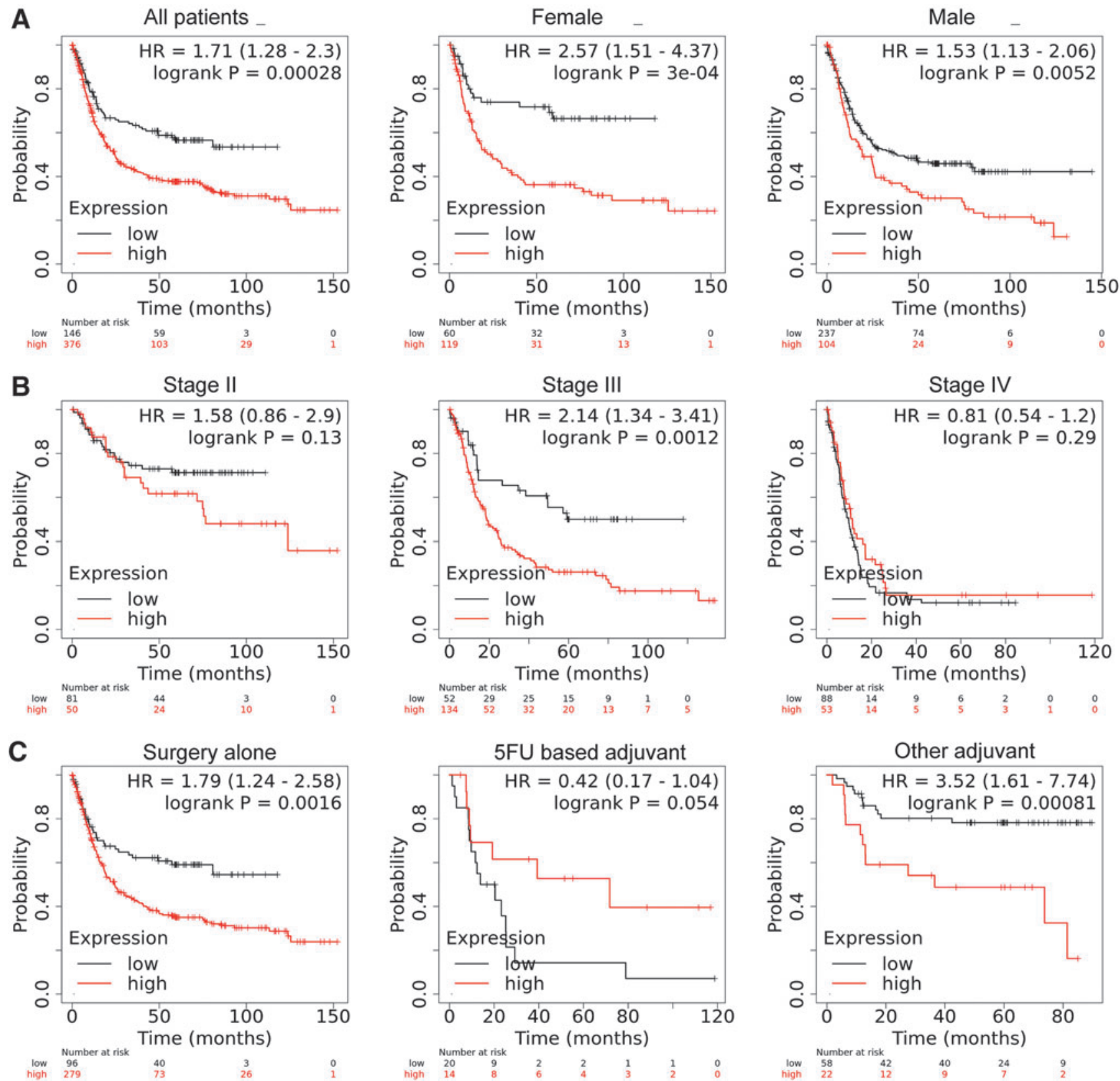

FIG. 3. Association between lncRNA-HEIH expression and FP of patients with GC. (A) High mRNA levels of HEIH were associated with lower FP in all GC patients (female or male). (B) High mRNA levels of HEIH were associated with shorter FP in stage III, but not in stage II and IV GC patients. (C) High mRNA levels of HEIH were associated with FP in GC patients who received single treatment with surgery and those who received additional adjuvant therapy. This effect was not noted in GC patients with 5FU-based adjuvant therapy. FP, first progression. Color images are available online.

expression was significantly associated with worse OS, FP, and PPS compared with that noted in patients with low HEIH expression. Taken together, these results indicated that GC patients with high HEIH expression levels exhibited lower OS, FP, and PPS, and that HEIH may be a poor prognostic factor associated with the survival of GC patients.

\section{Knockdown of HEIH inhibits cell proliferation of GC}

SRB assay indicated that the proliferative activity of MKN45 and AGS cells in both siRNA-1 and siRNA-2 groups was significantly reduced compared with that of the $\mathrm{NC}$ group ( $p<0.05$, Fig. 5A), which indicated that the interference of $\mathrm{HEIH}$ expression inhibited cell proliferation of GC cells.

\section{Knockdown of HEIH enhances oxaliplatin sensitivity of GC cells}

In both MKN45 and AGS cells treated with increasing concentrations of oxaliplatin, lncRNA-HEIH interference significantly reduced cell viability compared with that of the 
Post-progression survival
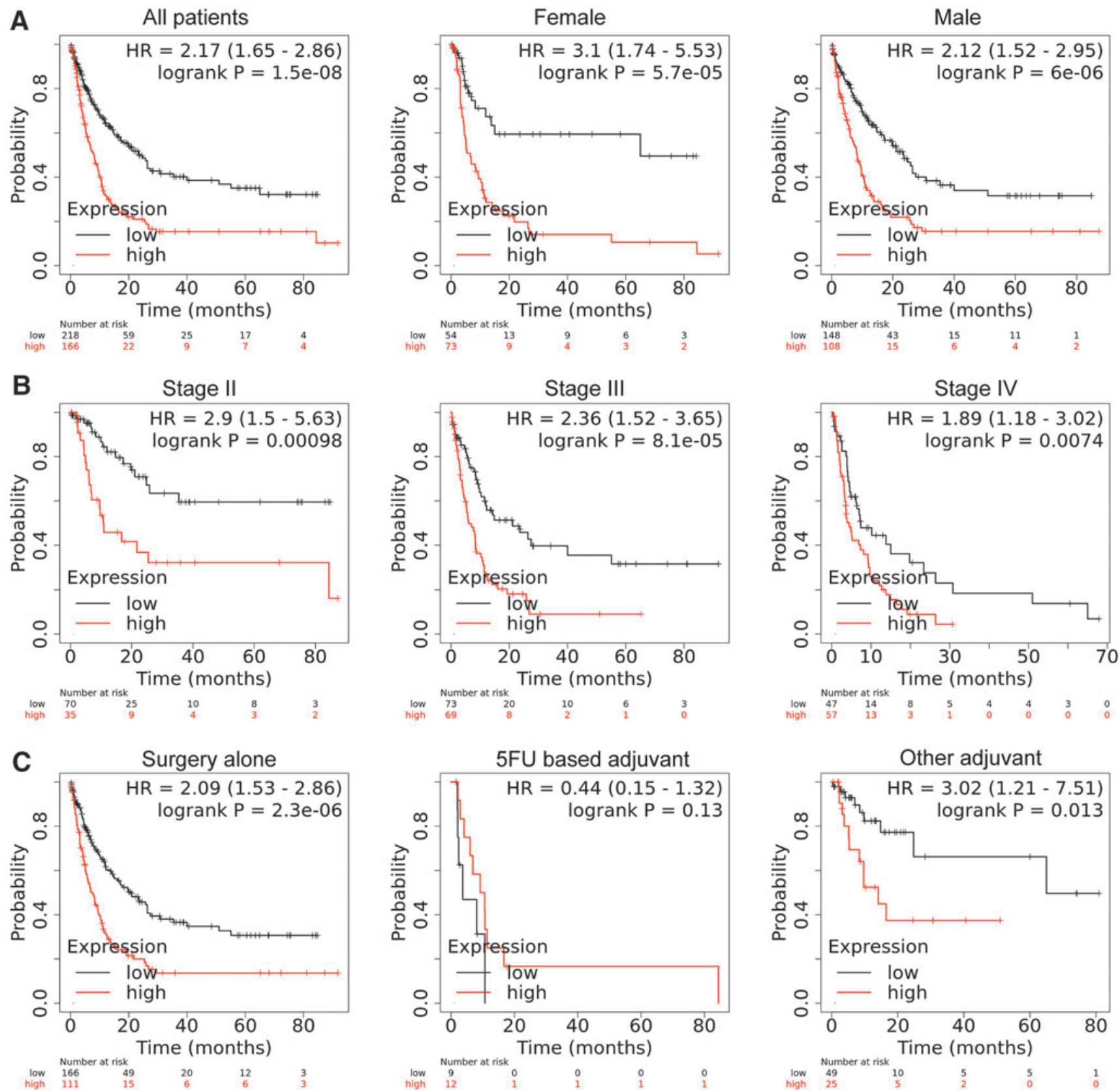

FIG. 4. Association between lncRNA-HEIH expression and PPS of patients with GC. (A) High mRNA levels of HEIH were associated with reduced PPS in all GC patients (female or male). (B) High mRNA levels of HEIH were associated with lower PPS in stage II, III, and IV GC patients. (C) High mRNA levels of HEIH were associated with PPS in GC patients who received single treatment with surgery and GC patients who received additional adjuvant therapy. This finding was not noted in GC patients who received 5FU-based adjuvant therapy. PPS, postprogression survival. Color images are available online.

siRNA groups $(p<0.05$; Fig. 5B). These results indicated that interference of HEIH expression could enhance oxaliplatin sensitivity in GC cells.

\section{Knockdown of HElH upregulates the expression of p53 in GC}

Following lncRNA-HEIH interference, the p53 expression levels of MKN45 and AGS cells were detected by RT-qPCR. The relative expression of p53 was significantly upregulated in the siRNA-HEIH group of GC cells compared with that of the NC groups (Fig. 5C, $p<0.05$ ). HEIH inhibited the expression of p53 in MKN45 and AGS cells, suggesting that it may be a downstream molecule of HEIH.

\section{Discussion}

GC has become a major threat to human health due to its late detection and poor prognosis (Van Cutsem et al., 2016). IncRNAs, which have limited protein-coding ability, 
FIG. 5. (A) SRB assay demonstrating that knockdown of IncRNA-HEIH inhibits cell proliferation ability of MKN45 and AGS cells. (B) The results from the SRB assay indicated that siRNA-1 or siRNA-2 GC cells exhibited increased sensitivity to treatment following an increase in the concentrations of oxaliplatin in MKN45 and AGS cells.

(C) P53 expression was upregulated following knockdown of lncRNA-HEIH in both MKN45 and AGS cells. $* p<0.05$ versus NC. SRB, sulforhodamine blue; siRNA1, siRNA-HEIH-1; siRNA-2, siRNA-HEIH-2; NC, negative control; siRNA, small interfering RNA. Color images are available online.
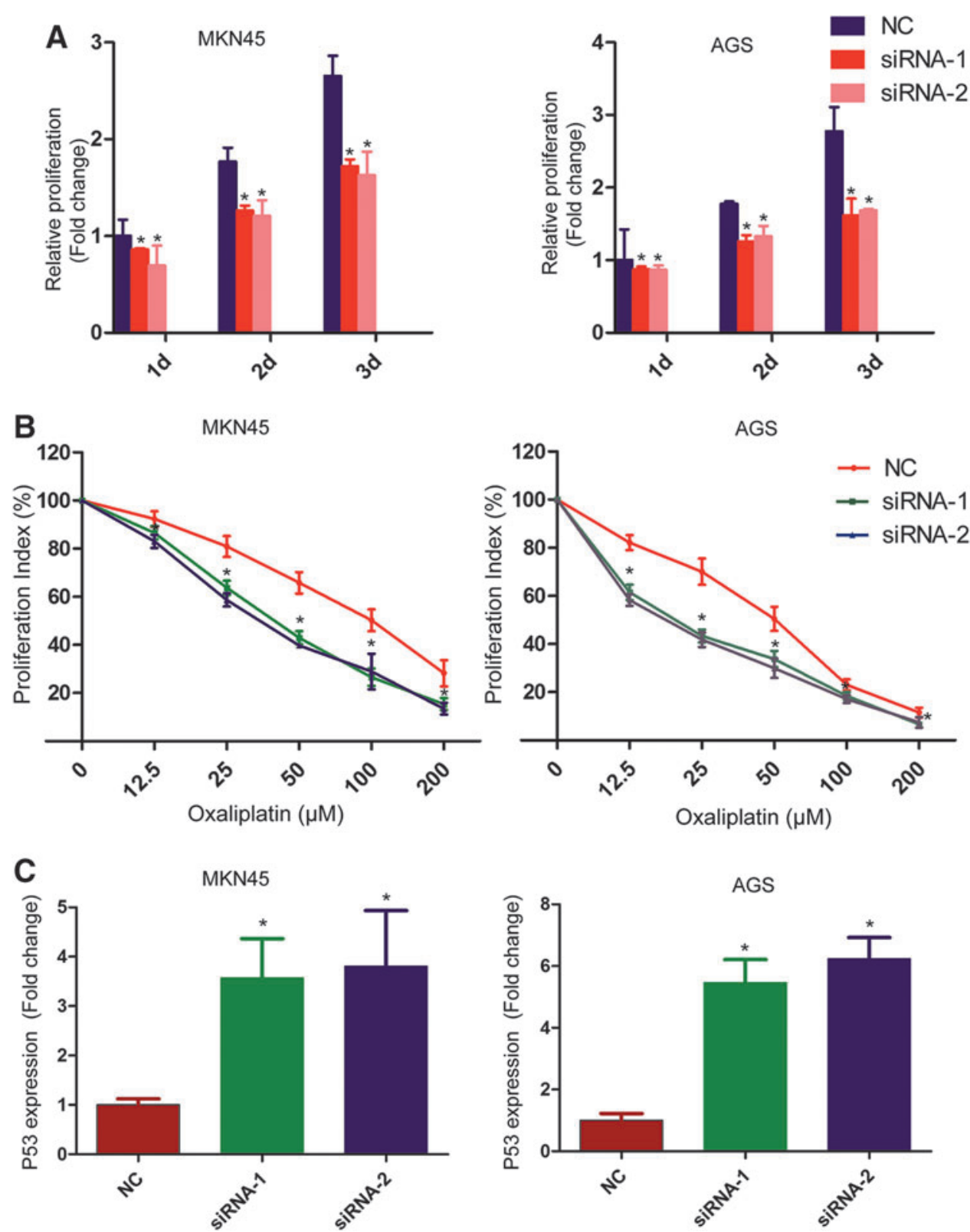

play critical roles in cancer progression and metastasis (Mirhosseini et al., 2019). IncRNA-based clinical tools have been rapidly developed, including diagnostic and prognostic biomarkers, as well as therapeutic targets (Mao et al., 2018; Yan et al., 2019; Zhang et al., 2019). Although the role of HEIH in diverse cancer types has been previously reported, the expression of this lncRNA and its clinical significance in $\mathrm{GC}$ remain unclear.

To the best of our knowledge, this study provides the first preliminary evidence to support the association between HEIH expression and GC. HEIH was highly expressed in human GC tissues and cell lines. A significant association between HEIH expression levels and specific clinical parameters was reported, including medium-high differentiation, distant metastasis, lymph node metastasis, and a larger tumor size. These results suggested the potential role of lncRNA-HEIH as a molecular biomarker of GC. Subsequently, the prognostic value of HEIH in GC was assessed. This study further assessed whether high HEIH expression could predict poor prognosis of GC patients by KM survival analysis. The results suggested that HEIH expression may aid the prediction of the postoperative tumor progression and were consistent with those of Cui et al. (2018). Therefore, lncRNAs may be used as markers for cancer diagnosis and prognosis.

A previous study has indicated that lncRNA-HEIH is involved in the modulation of tumor progression (Wan et al., 2020). To explore the role of HEIH in GC, its expression was knocked down by siRNAs in GC cells. SRB analysis indicated that HEIH knockdown significantly inhibited the viability of MKN45 and AGS cells. Several studies have previously demonstrated that lncRNAs are involved in drug resistance of GC (Yang et al., 2015; Ayers and Vandesompele, 2017; Heery et al., 2017). Guo et al. (2019) reported that downregulation of IncRNA-HEIH reversed paclitaxel resistance of endometrial cancer cells by activation 
of the MAPK signaling pathway. Based on these conclusions, the effect of HEIH expression was explored on oxaliplatin sensitivity in GC cells by the SRB assay. The data demonstrated that HEIH weakened chemotherapeutic sensitivity to oxaliplatin in GC cells. However, the precise molecular mechanism of this process requires further investigation.

P53 is widely accepted as one of the most important tumor suppressor proteins (Kruiswijk et al., 2015). Mutation and/or deletion of the p53 gene plays a prominent role across numerous malignancies, including GC (Lin et al., 2019; Blanchet et al., 2021). Increasing evidence has shown that IncRNAs are associated with the p53 pathway in a number of in vitro and in vivo models (Chen et al., 2020; Zhao et al., 2020). It was also shown that knockdown of lncRNA-HEIH downregulated the expression levels of p53, which suggested a possible lncRNA-mediated transcriptional regulation of $\mathrm{p} 53$.

The limitation of this study was the lack of in vivo experiments. Animal experiments may aid the clarification of the exact function and regulatory mechanisms of $\mathrm{HEIH}$ in $\mathrm{GC}$.

\section{Conclusion}

In conclusion, this study examined the role of lncRNA$\mathrm{HEIH}$ by validating its oncogenic and prognostic potential in GC. High HEIH expression was associated with poor prognosis in GC patients. Moreover, knockdown of HEIH exhibited antitumor properties by inhibition of cell viability, promotion of sensitivity to oxaliplatin, and induction of p53 expression in GC. These findings suggest the potential application of HEIH as a biomarker for the prognosis of GC. However, additional studies are required to verify the findings of this study.

\section{Authors' Contributions}

X.C. and X.S. conceived and designed the experiments. X.L., X.C., and X.S. performed all the experiments. W.Y. and L.X. wrote and revised the article. All authors read and approved the final version of the article.

\section{Author Disclosure Statement}

No competing financial interests exist.

\section{Funding Information}

This work was supported by the grants from Research Foundation of Jiangsu Provincial Natural Science Research Project. 2017.10-2019.10. (17KJD320003) and Research Foundation of Suzhou Youth Science and technology project. 2017.10-2020.10. (KJXW2017006).

\section{References}

Ayers D, Vandesompele J (2017) Influence of microRNAs and long non-coding RNAs in cancer chemoresistance. Genes (Basel) 8:95.

Blanchet A, Bourgmayer A, Kurtz JE, et al. (2021) Isoforms of the p53 family and gastric cancer: a menage a trois for an unfinished affair. Cancers (Basel) 13:916.

Chen S, Thorne RF, Zhang XD, et al. (2020) Non-coding RNAs, guardians of the p53 galaxy. Semin Cancer Biol 30: 190-195.
Cui C, Zhai D, Cai L, et al. (2018) Long noncoding RNA HEIH promotes colorectal cancer tumorigenesis via counteracting miR-939 mediated transcriptional repression of Bcl-xL. Cancer Res Treat 50:992-1008.

Fatica A, Bozzoni I (2014) Long non-coding RNAs: new players in cell differentiation and development. Nat Rev Genet 15:7-21.

Guo JL, Tang T, Li JH, et al. (2019) lncRNA HEIH enhances paclitaxel-tolerance of endometrial cancer cells via activation of MAPK signaling pathway. Pathol Oncol Res 26:1757-1766.

Heery R, Finn SP, Cuffe S, Gray SG (2017) Long non-coding RNAs: key regulators of epithelial-mesenchymal transition, tumour drug resistance and cancer stem cells. Cancers (Basel) 9:38.

Iyer MK, Niknafs YS, Malik R, et al. (2015) The landscape of long noncoding RNAs in the human transcriptome. Nat Genet 47:199-208.

Jia K, Chen F, Xu L (2019) Long noncoding RNA HEIH promotes the proliferation and metastasis of non-small cell lung cancer. J Cell Biochem 120:3529-3538.

Kruiswijk F, Labuschagne CF, Vousden KH (2015) p53 in survival, death and metabolic health: a lifeguard with a licence to kill. Nat Rev Mol Cell Biol 16:393-405.

Li P, Zhou B, Lv Y, Qian Q (2019) lncRNA HEIH regulates cell proliferation and apoptosis through miR-4458/SOCS1 axis in triple-negative breast cancer. Hum Cell 32:522-528.

Lin T, Hou PF, Meng S, et al. (2019) Emerging roles of p53 related lncRNAs in cancer progression: a systematic review. Int J Biol Sci 15:1287-1298.

Mao X, Qin X, Li L, et al. (2018) A 15-long non-coding RNA signature to improve prognosis prediction of cervical squamous cell carcinoma. Gynecol Oncol 149:181-187.

Mirhosseini SA, Sarfi M, Samavarchi Tehrani S, et al. (2019) Modulation of cancer cell signaling by long noncoding RNAs. J Cell Biochem 120:12224-12246.

Orellana EA, Kasinski AL (2016) Sulforhodamine B (SRB) assay in cell culture to investigate cell proliferation. Bio Protoc 6:e1984.

Rawla P, Barsouk A (2019) Epidemiology of gastric cancer: global trends, risk factors and prevention. Prz Gastroenterol 14:26-38.

Smyth EC, Nilsson M, Grabsch HI, et al. (2020) Gastric cancer. Lancet 396:635-648.

Spizzo R, Almeida MI, Colombatti A, Calin GA (2012) Long non-coding RNAs and cancer: a new frontier of translational research? Oncogene 31:4577-4587.

Thrift AP, El-Serag HB (2020) Burden of gastric cancer. Clin Gastroenterol Hepatol 18:534-542.

Van Cutsem E, Sagaert X, Topal B, et al. (2016) Gastric cancer. Lancet 388:2654-2664.

Wan T, Wang H, Gou M, et al. (2020) lncRNA HEIH promotes cell proliferation, migration and invasion in cholangiocarcinoma by modulating miR-98-5p/HECTD4. Biomed Pharmacother 125:109916.

Wang FH, Shen L, Li J, et al. (2019) The Chinese Society of Clinical Oncology (CSCO): clinical guidelines for the diagnosis and treatment of gastric cancer. Cancer Commun (Lond) 39:10.

Yan J, Zhou C, Guo K, et al. (2019) A novel seven-lncRNA signature for prognosis prediction in hepatocellular carcinoma. J Cell Biochem 120:213-223.

Yang F, Zhang L, Huo XS, et al. (2011) Long noncoding RNA high expression in hepatocellular carcinoma facilitates tumor growth through enhancer of zeste homolog 2 in humans. Hepatology 54:1679-1689. 
Yang Q, Zhang RW, Sui PC, et al. (2015) Dysregulation of noncoding RNAs in gastric cancer. World J Gastroenterol 21 10956-10981.

Zhang H, Zhu M, Du Y, et al. (2019) A panel of 12-lncRNA signature predicts survival of pancreatic adenocarcinoma. J Cancer 10:1550-1559.

Zhang PF, Sheng LL, Wang G, et al. (2016) miR-363 promotes proliferation and chemo-resistance of human gastric cancer via targeting of FBW7 ubiquitin ligase expression. Oncotarget 7:35284-35292.

Zhao H, Xing G, Wang Y, et al. (2017) Long noncoding RNA HEIH promotes melanoma cell proliferation, migration and invasion via inhibition of miR-200b/a/429. Biosci Rep 37: BSR20170682.

Zhao W, Jin Y, Wu P, et al. (2020) LINC00355 induces gastric cancer proliferation and invasion through promoting ubiquitination of P53. Cell Death Discov 6:99.

Zong L, Abe M, Seto Y, Ji J (2016) The challenge of screening for early gastric cancer in China. Lancet 388:2606.
Address correspondence to: Lu $X u, M D$

Department of General Surgery The First Affiliated Hospital of Soochow University

No. 899, Ping Hai Road

Suzhou 215006

China

E-mail: luxu@suda.edu.cn

Wenyan $Y u, M M$

Department of Oncology

The First Affiliated Hospital of Soochow University

No. 899, Ping Hai Road

Suzhou 215006

China

E-mail: $20175232181 @$ stu.suda.edu.cn 